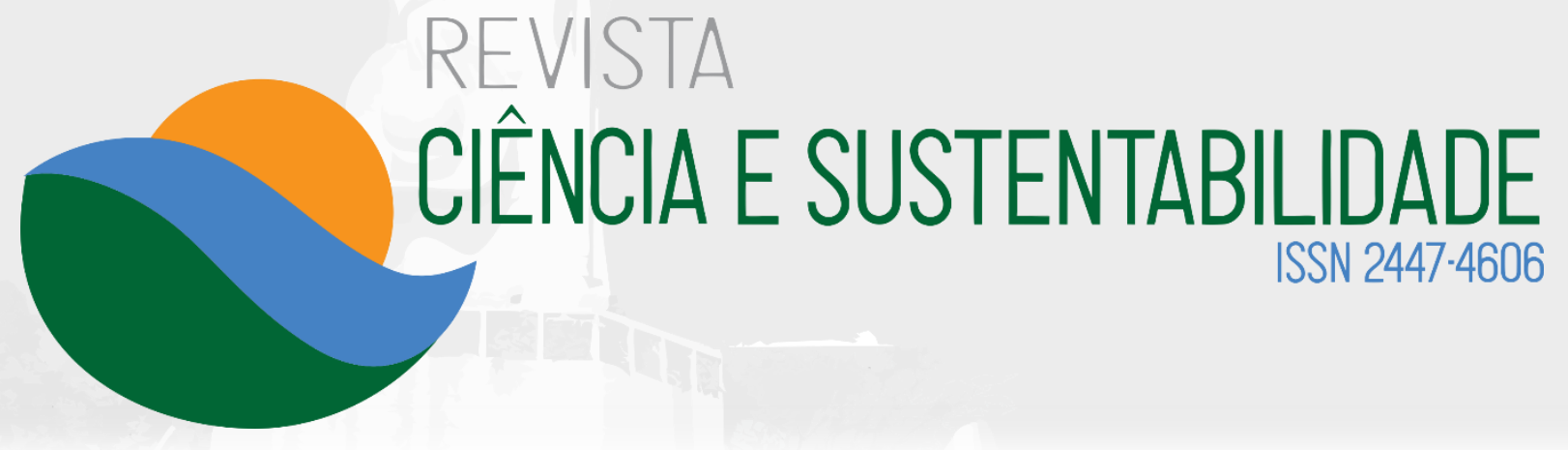

\title{
Desenvolvimento sustentável, sustentabilidade e saúde: uma revisão
}

\section{Sustainable development, sustainability and health: a review}

\section{Leandro Januário de Lima}

Universidade Federal de Campina Grande (UFCG) Graduando do Bacharelado em Ciências Médicas (Medicina) na Universidade Federal de Campina Grande (UFCG), campus Cajazeiras, E-mail: leandrojanuario100@gmail.com

\section{José Ferreira Lima Junior}

Universidade Federal de Campina Grande (UFCG), Graduação em Odontologia pela Universidade Federal do Rio Grande do Norte (UFRN) (2003); mestrado em Odontologia Preventiva e Social pela UFRN (2005) e doutorado em Biotecnologia em Saúde pela Universidade Federal da Paraíba - UFPB (2012). É docente da Universidade Federal de Campina Grande (UFCG), Email: proferjunior@gmail.com

\footnotetext{
Ysa Helena Diniz Morais de Luna Universidade Federal da Paraíba (UFPB), Graduação em Engenharia Ambiental pela Universidade Federal da Paraíba (2014), Mestre em Ciência e Tecnologia Ambiental pela Universidade Estadual da Paraíba (2017) na área de Saneamento Ambiental e Doutoranda em Saneamento Ambiental pelo Programa de Pós Graduação em Engenharia Civil e Ambiental pela Universidade Federal da Paraíba (2018), E-mail: ysa_luna@outlook.com
}

RESUMO

Objetivo: Este trabalho buscou revisar aspectos da relação entre Sustentabilidade e Ciências da Saúde, a partir da abordagem de áreas das instituições hospitalares que são permeadas pelo Desenvolvimento Sustentável. Material e Método: Trata-se de uma pesquisa exploratória, de natureza quantitativa, delineada como bibliográfica. Resultados e Discussão: Foram abordados os determinantes sociais do processo saúde-doença, a gestão de resíduos sólidos, práticas de gestão sustentáveis e as nuances ambientais na arquitetura e infraestrutura hospitalar. No estudo da relação entre Saúde e Meio Ambiente, nota-se uma relação intrínseca da degradação ambiental como determinante do padrão epidemiológico de emergência e reemergência das doenças. Quanto à questão dos resíduos sólidos, constatou-se que o arcabouço jurídico, administrativo já é suficiente à solução da problemática, contudo, falta efetivação e fiscalização. As práticas sustentáveis na área da gestão, que se relacionam principalmente com o pilar econômico do desenvolvimento sustentável, também impactam na sustentabilidade ambiental, pois, a melhoria da gestão dos recursos reduz a compra de excedentes e a produção de resíduos. 0 apelo para adoção de práticas sustentáveis também vem ganhando espaço na arquitetura hospitalar, avançando na preocupação com o impacto ambiental do prédio. Conclusão: Mesmo que inicialmente restrita às noções de determinação ambiental do processo saúde-doença, a Sustentabilidade vem ganhando espaço nos campos administrativos, de infraestrutura, de gestão de recursos, que por se moldarem às necessidades 
Desenvolvimento sustentável, sustentabilidade e saúde: uma revisão

Giliara Carol Diniz Gomes de Luna Universidade Federal de Campina Grande (UFCG), Graduação em Odontologia pela Universidade Federal da Paraíba (2004) e Mestrado em Odontologia (Diagnóstico Bucal) pela mesma instituição, obtido em 2007. Doutora em Odontologia pelo Programa Integrado de Pós-graduação em Odontologia UFPB-UFBA (2010). Atualmente compõe o corpo docente da Escola Técnica de Saúde de Cajazeiras (ETSC), no Centro de Formação de Professores(CFP) da UFCG, E-mail: giliaraluna@gmail.com

Recebido em: 08/11/2018 I Aceito em: $12 / 02 / 2018$ particulares do setor Saúde tornam-se estratégicos para a adoção de práticas baseadas no Desenvolvimento Sustentável.

Palavras-chave: Gestão Ambiental. Saúde Ambiental. Sustentabilidade.

\section{ABSTRACT}

Objective: This work sought to review aspects of the relationship between Sustainability and Health Sciences, from the approach of areas of hospital institutions that are permeated by Sustainable Development. Material and Method: It is an exploratory research, of quantitative nature, outlined as Bibliographic. Results and Discussion: The social determinants of the health-disease process, solid waste management, sustainable management practices and the environmental nuances in hospital architecture and infrastructure were addressed. In the study of the relationship between Health and the Environment, an intrinsic relation of the environmental degradation is set as determinant of the epidemiological pattern of diseases's emergency and reemergence. With regard to the issue of solid waste, it was found that the legal and administrative framework is sufficient to solve the problem, however, lack of enforcement and supervision. Sustainable management practices, which are mainly related to the economic pillar of sustainable development, also impact on environmental sustainability, since improving resource management reduces the purchase of surplus and the production of waste. The call for adoption of sustainable practices has also been gaining ground in thearchitectural design of buildings, advancing in the concern with the environmental impact of the building. Conclusion: Although initially restricted to the notions of environmental determination of the health-disease process, Sustainability has been gaining space in the administrative, infrastructure, and resource management fields, which by shaping the particular needs of the health sector become strategic for the the adoption of practices based on Sustainable Development.

Keywords: Environmental Administration. Environmental Health. Sustainability. 


\section{INTRODUÇÃO}

Provavelmente um dos termos mais citados nos últimos anos, o Desenvolvimento Sustentável (DS) teve suas bases lançadas no ano de 1713, com o lançamento da obra de Carlowitz, que trouxe o conceito inédito de nachhaltend ou nachhaltig - nascia o termo sustentável (FEIL; SCHREIBER, 2017), que implica na busca do equilíbrio entre as necessidades do ser humano e o meio ambiente, e em compreender o dinamismo da interação entre estes, a fim de aprofundar e ampliar seu significado (BARBOSA, DRACH e CORBELLA, 2014).

$O$ interesse pela temática se acentua no século XX e permanece até hoje, com a intensificação de abordagens que contemplam a produção de bens de forma limpa, controle de emissão de poluentes, consumo sustentável. Cada uma destas é fruto da particularidade das ciências que em sua base epistemológica se dedicam a porções do conceito (SARTORI; LATRÔNICO; CAMPOS, 2014).

Partindo do pressuposto que o DS permeia os diversos saberes e práticas, sua relação com os serviços de saúde torna-se um ponto de análise fundamental. Esta pode ser analisada sob a convergência dos pilares do DS: dimensões econômica, social e ambiental (OPAS, 2014).

Vários são os impactos na saúde humana decorrentes das atividades predatórias dirigidas ao meio ambiente (RATTNER, 2009), e esta dimensão embora recentemente seja estudada com mais afinco, traz à tona a a necessidade de analisar como as instituições e serviços de saúde pode contribuir para a promoção de uma sociedade mais sustentável.

Nos últimos anos, é crescente o interesse pela abordagem dos resíduos sólidos dos serviços de saúde (FREIRE; MOREIRA, 2016), cuja importância se justifica pelo alto consumo de produtos de uso único em estabelecimentos de saúde, que ainda são dotados de descarte específico, implicando em manejo inadequado dos resíduos e poluição do meio.

As atividades do setor de serviços geram impactos ambientais, que incluem o consumo de água e energia elétrica, geração de resíduos sólidos e efluentes líquidos, poluição do ar, configurando complexa interação com ambientes naturais ou 
urbanos, sob o escopo da sustentabilidade, a adoção de medidas para racionalização do emprego dos recursos naturais é capaz de desempenhar papel central na mitigação dos impactos socioambientais associados ao setor.

Neste sentido, este trabalho buscou revisar aspectos da relação entre Sustentabilidade e Ciências da Saúde, a partir da abordagem de áreas das instituições hospitalares que são permeadas pelo Desenvolvimento Sustentável.

\section{MÉTODO}

Trata-se de uma pesquisa exploratória, de natureza quantitativa, delineada como bibliográfica. Pode-se definir a pesquisa bibliográfica como aquela na qual a construção dos resultados para a pergunta de partida se dá a partir do conhecimento já sedimentado principalmente em livros e artigos científicos (GIL, 2017).

O caráter exploratório deste trabalho se assenta na abordagem ainda incipiente das intersecções entre o conceito de Desenvolvimento Sustentável e as atividades da área de Ciências da Saúde. Já a natureza qualitativa, foi escolhida por proporcionar a interpretação dos fenômenos colhidos e a atribuição de significado, que neste caso não são parâmetros que possam ser traduzidos em números (PRODANOV; FREITAS, 2013).

Foram seguidas, neste trabalho, as etapas da Pesquisa Bibliográfica descritas por Gil (2017): a) escolha do tema; b) levantamento bibliográfico preliminar; c) formulação do problema; d) elaboração do plano provisório de assunto; e) busca das fontes; f) leitura do material; g) fichamento; h) organização lógica do assunto; e i) redação do texto.

Como critérios de inclusão dos trabalhos, foram utilizados os que se seguem: estar no formato de livro, artigo publicado em periódico revisado por pares, documento oficial ou trabalho monográfico (Trabalho de Conclusão de Curso, Dissertação ou Tese); ter sido publicado nos últimos 20 anos. As fontes encontradas passaram por processo de leitura exploratória e posterior leitura analítica para redação do trabalho. 


\section{RESULTADOS E DISCUSSÃO}

\subsection{Saúde, Meio Ambiente e Sustentabilidade}

Desde o século passado quando se consolidou a ideia de História Natural da Doença, as análises epidemiológicas do processo saúde-doença passaram a evidenciar a relação entre homem e ambiente como uma das suas determinantes. No conceito introduzido por Leavell e Clarck que se dividia nos períodos pré-patogênico e patogênico, o primeiro é dirigido, sobretudo, às relações paciente suscetívelambiente. Os componentes destes fatores ambientais seriam a situação geográfica, solo, clima, relevo, agentes de natureza química ou física. Em situações de desequilíbrio resultantes destes componentes há uma perturbação que pode produzir quebra da homeostasia dentro de organismos vivos podendo funcionar como fenômenos patogênicos (ROUQUARIOL; ALMEIDA FILHO, 2003).

O processo saúde-doença é influenciado também por fatores sociais (socioeconômicos, sociopolíticos, socioculturais, psicossociais). Quando somados à falta de saneamento básico, acesso a alimentos com excesso de agrotóxicos, degradação do solo, do ar, das águas e nascentes, poluição por resíduos químicos letais oriundos de atividades como mineração e indústria petroquímica, entre outras condições de nosso tempo, têm-se graves ameaças à saúde humana (RATTNER, 2009). A relação saúde-meio ambiente se mostra recíproca pois, a boa saúde contribuí para o alcance de outras metas sociais como o desenvolvimento econômico e a preservação do meio ambiente, sendo apontada como componente inerente a todas as faces do desenvolvimento (CARVALHO, 2013).

As transformações no meio ambiente resultantes da atividade humana realizada de maneira degradante são responsáveis pela disseminação de novos e velhos agentes etiológicos alterando significativamente o padrão das doenças emergentes e reemergentes. As novas complexidades insurgentes no campo da Saúde Pública do século XXI derivam em grande parte dos ciclos epidêmicos resultantes de novos agentes etiopatogênicos - como o Vírus da Imunodeficiência Humana - e também do aumento na incidência de doenças cujo número de 
portadores tinha declinado - como dengue e leptospirose. Tanto na emergência quanto na reemergência de doenças na atualidade há uma relação com a degradação ambiental, desenvolvimento econômico e globalização (MINAYO; MIRANDA, 2002).

Os hospitais são os grandes expoentes da prestação de serviços no setor da saúde. No Brasil, em virtude de políticas hospitalocêntricas implantadas principalmente na segunda metade do século $X X$, são atualmente mais de 6300 hospitais compondo o Sistema Único de Saúde (SUS) e atendendo a uma população de mais de 200 milhões de habitantes (PAIM et al., 2011). Estes dados aliados às peculiaridades destes estabelecimentos que contam com atividades de grande potencial para a produção de resíduos como o seu funcionamento 24 horas durante 365 dias ao ano, e a alta demanda por água e recursos energéticos torna-os importantes eixos na implantação de uma cultura voltada ao desenvolvimento sustentável, sobretudo na sustentabilidade ambiental (TOLEDO; DEMAJOROVIC, 2006).

Logo, com a aplicação do conceito de ecoeficiência ao modelo de gestão hospitalar reduzir-se-á os impactos ao meio ambiente atacando as suas causas e não as consequências. A ecoeficiência é definida por Pizzorno; Uhlmann; Pfitscher (2013, p. 6), "como uma gestão através do qual se pode relacionar competitividade e desenvolvimento sustentável com objetivo de criar e promover resultados com o menor impacto ambiental possível". Assim, o aprimoramento do processo gerencial com a gestão ambiental nesta atividade com a aplicação da gestão ambiental faz com que os impactos ambientais gerados pelo setor da saúde possam ser amenizados culminando na sustentabilidade ambiental da área (PFITSCHER et al., 2007).

\subsection{Resíduos dos Serviços de Saúde (RSS)}

Resíduos sólidos são conceituados, a partir da Lei 12.305/2010 que institui a Política Nacional de Resíduos Sólidos, como resíduos nos estados sólidos e semissólidos, que resultam de várias atividades, sejam indústrias e/ou domésticas, sejam de saúde, agrícolas e de serviços (BRASIL, 2010). Também os lodos 
provenientes de sistemas de tratamento de água, aqueles originados em equipamentos e instalações de controle de poluição; bem como alguns tipos de líquidos, cujas particularidades tornem inviável o seu lançamento na rede pública de esgotos ou corpo d'água, ou exijam para isso soluções técnicas e economicamente inviáveis em face à melhor tecnologia disponível.

Podemos definir resíduos como sendo todo material proveniente de atividades humanas nas indústrias, comércios e residências que seja considerado inútil. Neste conceito, o termo lixo, está incluído sob as diversas formas, inclusive o lixo tóxico e prejudicial ao meio ambiente. Por resíduos sólidos, de acordo com sua composição química, teremos os resíduos orgânicos, composto de matéria viva, como por exemplo, restos de alimentos e dejetos humanos e os resíduos inorgânicos, composto de materiais fabricados pelo homem, tais como plástico, vidro e metal.

As unidades hospitalares brasileiras são responsáveis por $1 \%$ de todo 0 conjunto de resíduos sólidos coletados diariamente no Brasil, perfazendo um total de mais de 2.300 toneladas diárias (GARCIA; ZANETTI-RAMOS, 2004). Os resíduos sólidos dos serviços de saúde, também denominados lixo hospitalar ou RSS podem ser definidos como "rejeitos produzidos pelos estabelecimentos de saúde, abrangendo hospitais, clínicas veterinárias, farmácias, clínicas médicas e odontológicas, laboratórios de análises clínicas e outros" (DOI; MOURA, 2011, p. 339).

As atividades fins de um hospital são geradoras de um grande número de resíduos muitos deles descartáveis, que podem ou não estar infectados por agentes patogênicos. Entre aqueles do rol de elementos que podem ser infecciosos estão seringas e agulhas, curativos, embalagens de sangue e outros. As unidades hospitalares ainda são responsáveis pela produção de resíduos não infectantes de papelão, madeira, plásticos, tecidos e metais (TOLEDO; DEMAJOROVIC, 2006).

Desse modo, percebe-se que há grande diversidade de materiais nos RSS. Surge então uma preocupação ambiental que reside no fato que um erro durante as etapas do gerenciamento destes resíduos pode levar a um acidente ambiental afetando também a qualidade de vida da população que é dependente do equilíbrio e da sustentabilidade ambiental (COSTA; FONSECA, 2009). 
Se por um lado estudos mostram que a geração de RSS é rotineira desde os cuidados pré-hospitalares até o atendimento em alta complexidade, exemplificados, respectivamente, nos trabalhos de Mendes et al. (2015) e Rosa; Mthias; Komata (2015), por outro ${ }_{\llcorner}$a legislação para destinação final é descumprida por falta de planejamento e gestão dos resíduos sólidos em geral nos municípios. Ressalta-se que as ferramentas jurídicas disponíveis no Brasil já apresentam soluções para o manejo dos resíduos sólidos, embora ainda careçam de efetivação e fiscalização (MORAES, 2016).

Como os RSS possuem natureza diversa e heterogênea é preciso classificar os resíduos para que haja uma segregação segura para o trabalhador e o Meio Ambiente. No Brasil foram propostas diferentes classificações para os RSS, as principais foram recomendadas pelo Conselho Nacional de Meio Ambiente (CONAMA) e pela Agência Nacional de Vigilância Sanitária (ANVISA), que por sua vez, através da resolução 33/2003 propôs uma classificação em cinco grupos para resíduos de saúde: Grupo A - potencialmente infectantes; Grupo B - químicos; Grupo C - rejeitos radioativos; Grupo D - resíduos comuns; e Grupo E - perfurocortantes (BRASIL, 2003). Atualmente vigora a RDC 222/2018 que regulamenta as Boas Práticas de Gerenciamento dos Resíduos de Serviços de Saúde e dá outras providências, além de trazer a classificação dos RSS mantendo -os mesmos grupos da RDC-306/2004 (BRASIL, 2004; BRASIL, 2018).

O CONAMA apresentou sua primeira classificação dos RSS em 1993 com a Resolução n5 do mesmo ano, atualizando-a depois em 2003. Esta classificação se constituiu na base para a proposta da ANVISA na década seguinte (GARCIA; ZANETTI-RAMOS, 2004). Segundo o CONAMA, os RSS deveriam ser agrupados em quatro grandes conjuntos: no grupo $A$, os que apresentassem grande risco ao Meio Ambiente e à população (matérias perfurocortantes ou contaminados); no grupo $B$, resíduos químicos; grupo $C$, rejeitos radioativos; e no grupo 4, resíduos comuns (BRASIL, 1993).

Atualmente a classificação obedece aos critérios da resolução 358 do CONAMA editada em 2005, substituindo a anterior. Nela, os RSS são classificados em cinco grupos: $A, B, C, D$ e $E$. Enquadram-se no grupo $A$ os que apresentam risco potencial à saúde pública e ao Meio Ambiente devido à possível presença de agentes Ciência e Sustentabilidade - CeS. v. 4, n. 2, p. 133-150, jul./dez. 2018 
biológicos, dentre eles, materiais contendo secreções e líquidos orgânicos; no grupo $B$ encontram-se os resíduos químicos; no grupo $C$ estão os rejeitos radioativos; no grupo $D$, os resíduos comuns e, no grupo $E$ os materiais perfurocortantes ou escarificantes, tais como agulhas e escalpes (BRASIL, 2005).

A partir da apresentação das diversas classificações para os RSS é possível perceber que mesmo com regulamentações que existem desde o final do século $X X$, ainda há um longo caminho a percorrer na segregação e destinação dos RSS de maneira sustentável. Reis et al. (2013) ressaltam que mesmo com a classificação muitos estabelecimentos de saúde desconhecem a quantidade e a composição dos resíduos que produzem. Este cenário resulta em manejo inadequado que oferece riscos ao meio ambiente e à saúde dos trabalhadores do setor. Como a produção de resíduos esbarra no desafio de como gerenciá- $\operatorname{los}_{2}$ haja vista que a existência humana é intrinsecamente ligada ao seu acúmulo, as organizações, inclusive as hospitalares, devem instituir ferramentas que garantam a sustentabilidade ambiental deste setor para evitar maiores danos ao planeta (FREIRE; MOREIRA, 2016).

\subsection{Práticas Sustentáveis na Gestão de Organizações Hospitalares}

Até o momento, a problemática da sustentabilidade ambiental nas organizações hospitalares foi tratada principalmente no nível do Estado, destacandose a sua função reguladora e norteadora do processo decisório. Esta análise parte do conceito de governabilidade que é o modo pelo qual o poder é exercido na administração dos recursos sociais e econômicos visando o desenvolvimento. No entanto, às práticas organizacionais internas são igualmente decisivas na busca da ampliação das atividades sustentáveis. Governo, sociedade e organizações devem atuar simultaneamente para que seja alcançado a nível local e global o desenvolvimento sustentável (BUSS et al., 2012).

As organizações modernas desenvolvem suas atividades geralmente pautadas nos princípios ou funções administrativas que são 4 a saber: planejamento, organização, direção e controle. A partir do planejamento a organização é capaz de determinar de maneira antecipada o que se deve fazer. Na função administrativa da 
organização, a entidade aloca seus recursos e determina sua estrutura administrativa. Por meio da função de direção são manuseados os recursos humanos para o cumprimento das funções administrativas anteriores. E durante a execução da função de controle ${ }_{\iota}$ o desempenho é comparado aos objetivos traçados pela organização para saber se estes estão sendo alcançados (CHIAVENATO, 2003; MAXIMIANO, 2000).

A pequena apresentação das funções administrativas abre um horizonte de potencialidades para implantação de ações sustentáveis dentro do processo administrativo. Algumas destas inovações já estão registradas na literatura, voltando-se predominantemente à dimensão social do desenvolvimento econômico. Sobretudo, por conta da instabilidade no orçamento público a implantação de modelos de gestão e contabilidade sustentável vem sendo utilizados para garantir o mínimo de qualidade e capacidade de resposta dos serviços (CAMPOS; RAMOS, 2014).

Para alcançar os objetivos traçados de maneira sustentável as organizações optam por investir em inovações administrativas, destacando-se à inovação nos processos que acaba por melhorar a eficiência, pois, interfere diretamente no processo produtivo (SILVA et al., 2012). Com base nos indicadores disponíveis para mensurar os impactos da sustentabilidade as organizações podem realizar a função administrativa de controle das atividades. Como nos últimos anos acentuou-se a implantação de medidas sustentáveis, tais indicadores vêm sendo revisados para oferecer dados ainda mais consistentes aos gestores (VEIGA, 2010).

As inovações nos processos dentro dos ambientes hospitalares estão surgindo muitas vezes como mudanças pontuais, mas que conseguem contribuir com a sustentabilidade ambiental das organizações. O exemplo mais comum vem sendo a redução do uso de papel mediante a implantação de prontuários eletrônicos. Muito embora o uso de sistemas eletrônicos acabe aumentando o consumo energético nas unidades hospitalares que já são grandes consumidoras, a redução do uso de papel ajuda a reduzir o desmatamento de áreas florestais além de economizar a água que é consumida no processo produtivo. A utilização de serviços em nuvem aumenta a segurança dos dados do hospital e também reduz o número e o tamanho dos arquivos físicos (SILVA; PONTES, 2016; FREIXO; ROCHA, 2014). 
Outro setor dos hospitais que vem ganhando práticas sustentáveis é a farmácia onde ocorrem os processos de medicação. Esses processos são geradores de muitos RSS. Uma das primeiras inovações no setor foi o uso de softwares que além dos benefícios na redução do uso do papel ajudam na gestão da dispensação de medicamentos e no controle do estoque, favorecendo um regime de compras mais sustentável, pois, os fármacos são resíduos de impactos preocupantes no ambiente por sua capacidade de modificarem o metabolismo de organismos vivos. 0 treinamento das equipes de serviço para adoção de atitudes sustentáveis também é uma estratégia que auxilia na redução da produção de RSS mediante consumo sustentável dos materiais disponíveis (FURUKAWA et al., 2016).

As modificações na estrutura física dos hospitais para conciliar recursos terapêuticos de alta tecnologia com a otimização de recursos têm resultado em redução dos impactos ambientais, graças a projetos de gestão embasados na promoção da saúde com enfoque na sustentabilidade (URIBE; ARBOLEDA, 2015).

O processo de passagem para as práticas sustentáveis nas organizações hospitalares passa necessariamente pela construção de uma mentalidade voltada à sustentabilidade nos seus colaboradores. Com este mecanismo participativo que interfere diretamente nas interconexões existentes entre os recursos da organização é possível encontrar formas saudáveis e sustentáveis de atuar (SVALDI; SIQUEIRA, 2010).

\subsection{Arquitetura, Estrutura Física Hospitalar e Sustentabilidade}

Os hospitais são locais que primam pela saúde e bem-estar da população, logo, a presença da sustentabilidade desde a concepção do projeto de uma unidade de atenção à saúde deve ser uma constante (ROBERTO; CAVA, 2015).

A sustentabilidade vem ganhando espaço também na arquitetura dos empreendimentos. Por esta via o programa do empreendimento é relacionado às características locais, condicionantes, e recursos naturais disponíveis e o projeto é direcionado para uma convivência harmoniosa com a comunidade local. A sustentabilidade ambiental é contemplada a partir de diretrizes para os projetos 
preocupadas com os resíduos a serem produzidos durante a construção, o uso de água, a presença de efluentes e o gasto energético (ASBEA, 2012). Estas preocupações são universais e se estendem à construção de unidades hospitalares.

Portanto, é perceptível que as edificações sustentáveis são dotadas de práticas no projeto e na construção que buscam minimizar seu impacto no meio ambiente. $\mathrm{O}$ conceito de edificação ecológica estaria ligado à certificação por órgãos governamentais ou organizações competentes. Nos projetos hospitalares da chamada arquitetura bioclimática os assuntos ligados à estética da edificação ficam em segundo plano. 0 foco torna-se os aspectos funcionais do edifício, como a construção e os custos de manutenção (ZAMPIVA, 2016).

A arquitetura hospitalar também é subordinada aos conceitos consagrados na bibliografia como flexibilidade, expansividade, manutenção e humanização. A expansividade é uma das características destas construções, com aumento no número de pavimentos ou na própria área construída. Como estas edificações são grandes consumidores de água estão sendo constantemente implementadas fontes de captação alternativa além da indicação de paisagismo com árvores nativas que acaba reduzindo o uso de água na jardinagem (LOBO, 2010).

O projeto para a construção de um novo hospital que inclua conceitos de sustentabilidade tem seu custo. Sobretudo no Brasil, em que boa parte dos estabelecimentos de saúde são estatais, este é um parâmetro importante a considerar. Contudo, nos edifícios do Sistema Único de Saúde o custo inicial pode ser compensado pelas economias decorrentes do uso mais racional dos equipamentos e espaços. Os projetos podem propor diversas estratégias para obter sustentabilidade. As ações propostas geralmente caminham na direção do seguinte rol:

a) estratégias de acondicionamento do lugar: disposição das atividades conforme orientação espacial; captação e reuso das águas da chuva, entre outras atividades;

b) estratégias de resfriamento: promover ventilação natural; restringir ganhos solares; promover o resfriamento evaporativo; e, promover o resfriamento por radiação em locais com elevadas amplitudes térmicas; 
c) estratégias de iluminação natural: uso de vedações transparentes modulares, protegidas da radiação; prateleiras de luz, forros claros e/ou Vidros seletivos;

d) estratégias de eficiência energética: adquirir equipamentos de baixo consumo elétrico e de água; fazer o controle individual dos equipamentos e sistemas de iluminação; e, incorporar a vegetação no isolamento do edifício;

Ao aplicar muitas destas ações de gestão as instituições geralmente se submetem a processos de certificação para obterem o reconhecimento de suas práticas sustentáveis. Em alguns casos o controle sobre os indicadores de sustentabilidade começa logo na fase de construção do edifício, baseando-se em parâmetros de eco construção e gestão que avaliam se o canteiro de obras funciona com baixo impacto ambiental apresentando destinação correta e baixa produção de resíduos bem como eficiência energética e boa gestão da água (OLIVEIRA; SPOSTO; BLUMENSCHEIN, 2012).

Vários são os sistemas para certificação de sustentabilidade das construções. LEED, BREEAM e AQUA são apenas alguns exemplos. A certificação Leadership in Energy and Environmental Design (LEED) surgiu em 1993 e avalia a sustentabilidade por meio de seis categorias: localização e transportes; espaços sustentáveis; uso racional da água; energia e atmosfera; materiais e recursos; e, qualidade ambiental interna. O modelo brasileiro Alta Qualidade Ambiental (AQUA) avalia o projeto como um todo a partir de três exigências: criação de um ambiente interno saudável e confortável para os usuários; controle dos impactos da construção sobre o meio ambiente externo; e, preservação dos recursos naturais por meio da otimização de seu uso (ZAMPIVA, 2016).

\section{CONSIDERAÇÕES FINAIS}

Com o destaque midiático ao qual vem sendo exposto o debate das questões ambientais, o tema vem se tornando mais comum nas análises das diversas áreas do saber. Constatou-se durante a construção deste trabalho que a abordagem das questões ambientais já vem sendo levantada dentro das Ciências da Saúde, embora que ainda de forma incipiente. 
Mesmo que inicialmente restrita às noções de determinação ambiental do processo saúde-doença, a Sustentabilidade vem ganhando espaço nos campos administrativos, de infraestrutura, de gestão de recursos, que por se moldarem às necessidades particulares do setor Saúde tornam-se estratégicos para a adoção de práticas baseadas no Desenvolvimento Sustentável.

Logo, além de despertar o debate em setores pontuais é preciso alargar o alcance, permitindo que novas atividades-meio e atividades-fim na área da Saúde possam planejar e fomentar o desenvolvimento sustentável, de modo a permitir condições para um futuro cada vez mais próximo de uma sociedade verde.

Os autores declaram não haver nenhum conflito de interesses com a temática abordada neste estudo.

\section{REFERÊNCIAS}

ASBEA. Associação Brasileira dos Escritórios de Arquitetura. Guia sustentabilidade na arquitetura: diretrizes de escopo para projetistas e contratantes. São Paulo: Prata Design, 2012.

BARBOSA, G. S.; DRACH, P. R.; CORBELLA, O. D. A Conceptual Review of the Terms Sustainable Development and Sustainability. International Journal of Social Sciences, v. III, n. 2, 2014.

BRASIL. Lei 12.305/2010. Política Nacional de Resíduos Sólidos. Diário Oficial da União, Brasília, DF, 2 ago. 2010.

BRASIL. Resolução Rdc n 33, de 25 de fevereiro de 2003. Ministério da Saúde. Agência Nacional de Vigilância. Dispõe sobre o Regulamento Técnico para o gerenciamento de resíduos de serviços de saúde. Diário Oficial da União. Brasília, 05 mar. 2003.

BRASIL. Resolução n 358, de 29 de abril de 2005. Ministério do Meio Ambiente. Conselho Nacional do Meio Ambiente (CONAMA). Dispõe sobre o tratamento e a disposição final dos resíduos dos serviços de saúde e dá outras providências. Diário Oficial da União. Brasília, 04 maios 2005.

BRASIL. Resolução 05, de 5 de agosto de 1993. Ministério do Meio Ambiente. Conselho Nacional do Meio Ambiente (CONAMA). Dispõe sobre o plano de gerenciamento, tratamento e destinação final de resíduos sólidos de serviços de saúde, portos, aeroportos, terminais rodoviários e ferroviários. Diário Oficial da União. Brasília, 31 ago. 1993. 
BRASIL. Resolução n 306, de 07 de dezembro de 2004. Dispõe sobre o

Regulamento Técnico para o gerenciamento de resíduos de serviços de saúde.

Diário Oficial da União. Brasília, 2004.

BRASIL. Resolução n 222, de 28 de março de 2018. Regulamenta as Boas Práticas de Gerenciamento dos Resíduos de Serviços de Saúde e dá outras providências.

Diário Oficial da União. Brasília, 2018.

BUSS, P. M. et al. Governança em saúde e ambiente para o desenvolvimento sustentável. Ciência \& Saúde Coletiva, Rio de Janeiro, v. 17, n. 6, p. 1479-1491, jun., 2012.

CAMPOS, F. J. B.; RAMOS, H. R. Aplicação do Modelo Triple Bottom Line em um Hospital Público. Revista de Gestão Ambiental e Sustentabilidade - GeAS, v. 3, n. 1, p. 124-138, 2014.

CARVALHO, A. I. Determinantes sociais, econômicos e ambientais da saúde. In: FUNDAÇÃO OSWALDO CRUZ. A saúde no Brasil em 2030 - prospecção estratégica do sistema de saúde brasileiro: população e perfil sanitário. Rio de Janeiro: Fiocruz/Ipea/Ministério da Saúde/Secretaria de Assuntos Estratégicos da Presidência da República, 2013. Vol. 2. pp. 19-38.

CHIAVENATO, I. Introdução à teoria geral da administração: uma visão abrangente da moderna administração das organizações. 7. ed. Rio de Janeiro: Elsevier, 2003.

COSTA, W. M.; FONSECA, M. C. G. A importância do gerenciamento dos resíduos hospitalares e seus aspectos positivos para o meio ambiente. Hygeia: Revista Brasileira de Geografia Médica e da Saúde, v. 5, n. 9, p. 12- 31, dez., 2009.

DOI, K. M.; MOURA, G. M. S. S. Resíduos sólidos de serviços de saúde: uma fotografia do comprometimento da equipe de enfermagem. Revista Gaúcha de Enfermagem, Porto Alegre, v. 32, n. 2, p. 338-344, jun., 2011.

FEIL, A. A.; SCHREIBER, D. Sustentabilidade e desenvolvimento sustentável: desvendando as sobreposições e alcances de seus significados. Cad. EBAPE.BR, Rio de Janeiro, v. 15, n. 3, p. 667-681, Jul., 2017.

FREIRE, E. A.; MOREIRA, M. R. C. prospecção científica sobre resíduos de serviços de saúde na área de Ciências Ambientais. Ciência e Sustentabilidade - CeS, Juazeiro do Norte, v. 2, n. 1, p. 7-22, jan/jun, 2016.

FREIXO, J.; ROCHA, Á. Arquitetura de Informação de Suporte à Gestão da Qualidade em Unidades Hospitalares. Revista Ibérica de Sistemas e Tecnologicas de Informação, Porto, n. 14, p. 1-15, dez. 2014. 
FURUKAWA, P. O. et al. Environmental sustainability in medication processes performed in hospital nursing care. Acta Paulista de Enfermagem, v. 29, n. 3, p. 316$324,2016$.

GARCIA, L. P.; ZANETTI-RAMOS, B. G. Gerenciamento dos resíduos de serviços de saúde: uma questão de biossegurança. Cadernos de Saúde Pública, Rio de Janeiro, v. 20, n. 3, p. 744-752, jun., 2004.

GIL, A. C. Como elaborar Projetos de Pesquisa. 6. Ed. São Paulo: Atlas, 2017.

LOBO, A. V. R. Ferramenta de avaliação de sustentabilidade ambiental em edificações hospitalares na região metropolitana de Curitiba. 2010. 269 f. Dissertação (Mestrado em Construção Civil) - Curso de Construção Civil, Universidade Federal do Paraná, Curitiba, 2010.

MAXIMIANO, A. C. A. Introdução à Administração. 5. ed. São Paulo: Atlas, 2000.

MENDES, A. A. et al. Resíduos de serviços de saúde em serviço de atendimento préhospitalar móvel. Revista Brasileira de Enfermagem, Brasília, v. 68, n. 6, p. 11221129, dez. 2015.

MINAYO, M. C. S.; MIRANDA, A. C. (Orgs.). Saúde e ambiente sustentável: estreitando nós. Rio de Janeiro: Editora FIOCRUZ, 2002. 344 p.

MORAES, J. L. Planejamento e gestão de resíduos sólidos: um desafio aos municípios. Ciência e Sustentabilidade - CeS, Juazeiro do Norte, v. 2, n. 2, p. 63-83, jul./dez. 2016.

OLIVEIRA, J. A. C.; SPOSTO, R. M.; BLUMENSCHEIN, R. N. Ferramenta para avaliação da sustentabilidade ambiental na fase de execução de edifícios no Distrito Federal. Gepros: Gestão da Produção, Operações e Sistemas, v. 7, n. 2, p. 11-21, abr./jun.,2012.

OPAS. Organização Pan-americana de Saúde. Desenvolvimento Sustentável e Saúde: tendências dos indicadores e desigualdades no Brasil. Brasília, DF: OPAS, 2014. 30 p.

PAIM, J. et al. The Brazilian health system: history, advances, and challenges. The Lancet, v. 377, n. 9779, p. 1778-1797, 2011.

PFITSCHER, E. D. et al. A situação dos hospitais quanto ao gerenciamento dos aspectos e impactos ambientais. Cadernos EBAPE. BR, v. 5, n. 3, p. 1-18, set., 2007.

PIZZORNO, C. E. A.; UHLMANN, V. O.; PFITSCHER, E. D. Sustentabilidade ambiental no contexto hospitalar: estudo em um hospital do Rio Grande do Sul. RAHIS Revista de Administração Hospitalar e Inovação em Saúde, v. 10, n. 3, p. 1-16, set./dez., 2013. 
PRODANOV, C. C.; FREITAS, E. C. Metodologia do trabalho científico: métodos e técnicas da pesquisa e do trabalho. 2. ed. Novo Hamburgo: Feevale, 2013.

RATTNER, H. Meio ambiente, saúde e desenvolvimento sustentável. Ciência \& saúde coletiva, Rio de Janeiro, v. 14, n. 6, p. 1965-1971, dez., 2009.

REIS, M. A. et al. Conhecimento, prática e percepção sobre o gerenciamento de resíduos de serviços de saúde em estabelecimentos médicos veterinários de Salvador, Bahia. Revista Brasileira de Saúde e Produção Animal, Salvador, v. 14, n. 2, p. 287-298, jun., 2013.

ROBERTO, H. F. F.; CAVA, A. M. L. Hospital sustentável ambientalmente: reflexões para a gestão do projeto. Revista Acreditação: ACRED, v. 5, n. 9, p. 114-132, 2015.

ROSA, C. D. P.; MATHIAS, D.; KOMATA, C. C. Custo de Gerenciamento de Resíduos de Serviços de Saúde (RSS): Estudo de Caso da Unidade de Terapia Intensiva de Infectologia de Um Hospital Público em São Paulo. Revista de Gestão Ambiental e Sustentabilidade, v. 4, n. 2, p. 127-143, 2015.

ROUQUARIOL, M. Z.; ALMEIDA FILHO, N. Epidemiologia \& Saúde. 6. ed. Rio de Janeiro: MEDSI, 2003.

SARTORI, Simone; LATRONICO, Fernanda; CAMPOS, Lucila M.S. Sustentabilidade e desenvolvimento sustentável: uma taxonomia no campo da literatura. Ambient. soc., São Paulo, v. 17, n. 1, p. 01-22, Mar. 2014.

SILVA, C. L. et al. Inovação e sustentabilidade. Curitiba: Aymará Educação, 2012.

SILVA, J. L. C.; PONTES, T. B. Utilização de nuvem privada em ambiente corporativo como estratégia para sustentabilidade nas empresas públicas. Ciência e

Sustentabilidade - CeS, Juazeiro do Norte, v. 2, n. 2, p. 146-182, jul./dez., 2016.

SVALDI, J. S. D.; SIQUEIRA, H. C. H. Ambiente hospitalar saudável e sustentável na perspectiva ecossistêmica: contribuições da enfermagem. Escola Anna Nery, Rio de Janeiro, v. 14, n. 3, p. 599-604, set., 2010.

TOLEDO, A. F.; DEMAJOROVIC, J. Atividade hospitalar: impactos ambientais e estratégias de ecoeficiência. INTERFACEHS - Revista de Gestão Integrada em Saúde do Trabalho e Meio Ambiente, v.1, n.2, p. 1-23, dez., 2006.

URIBE, D. F; ARBOLEDA, A.F. Centros especializados de San Vicente Fundación: hospital verde con certificación leed. Revista Ingeniería Biomedica, Medellín, v. 9, n. 18, p. 51-56, dez., 2015.

VEIGA, J. E. Indicadores de sustentabilidade. Estudos avançados, São Paulo, v. 24, n. 68, p. 39-52, 2010. 
Desenvolvimento sustentável, sustentabilidade e saúde: uma revisão

ZAMPIVA, P. M. Hospitais mais sustentáveis: relações entre o meio ambiente construído, a assistência aos pacientes e os preceitos de sustentabilidade. 2016. 155 f. Dissertação (Mestrado Profissional) - Mestrado em Arquitetura e Urbanismo, Universidade do Vale do Rio dos Sinos, São Leopoldo, 2016. 